\title{
Are two views better than one? Investigating three-quarter view
}

\section{facial composites.}

Hayley Ness 1, Peter J.B Hancock 2, Leslie Bowie 3, Vicki Bruce 4 \& Graham Pike 1

In press, Journal of Forensic Practice

1: Dept of Psychology, Open University, Walton Hall, Milton Keynes, MK7 6AA

2: Dept of Psychology, University of Stirling, Stirling, FK9 4LA

3: ABM (UK) Ltd, Prospect House, 3 Padge Road, Boulevard Ind. Park, Beeston, Nottingham, NG9 2JR

4: School of Psychology, Newcastle University, Ridley Building 1, Queen Victoria Road, Newcastle Upon Tyne, NE1 7RU

Email: hayley.ness@open.ac.uk

Acknowledgements:

We would like to thank Krista Overliet and Sivakumar Anandaciva who assisted with the data collection for experiments one, two and four. We would also like to thank Michael Bromby, formerly of ABM UK for his technical assistance with PROfit and Duncan Robb and Jane Turnbull at Queen Margaret University College, Edinburgh for their assistance with the recruitment of participants.

\section{Abstract}

Purpose - The introduction of a new three-quarter-view female database in PROfit has enabled a careful consideration of view effects in facial composite construction. This article formally examines the impact of constructing full-face and three-quarter view composites under different encoding conditions. It also examines three-quarter view composites that have been automatically generated. Finally, this article investigates whether there is an identification benefit for presenting a full-face and three-quarter composite together.

Design/methodology/approach - The paper presents results from three experiments that examine the impact of encoding conditions on composite construction as well as the presentation of composites at the evaluation stage.

Findings - The results revealed that while standard full-face composites perform well when all views of the face have been encoded, care should be taken when a person has only seen one view. When a witness has seen a side view of a suspect, a three-quarter-view composite should be constructed. In addition, it would be beneficial for a witness to construct two composites of a suspect, one in full-face view and one in a three-quarter-view, particularly when the witness has only seen one view. Originality/value - No research to date has examined the impact of viewpoint in facial composite construction.

Keywords facial composite, three-quarter view, viewpoint, PROfit, eyewitness memory, forensic cognition

Paper type Research paper 


\section{Introduction}

Computerised composite systems assist a witness in constructing a facial likeness of a suspect. In the UK the E-FIT and PROfit systems are generally used, although older systems are available (e.g. FACES, Mac-A-Mug Pro) as well as newer, more sophisticated systems such as Evo-FIT (Frowd, Pitchford, Bruce, Jackson., et al, 2010) and E-FIT-V (George, Gibson, Maylin and Solomon, 2008).

Research has clearly demonstrated that computerised systems produce better likenesses than the older systems such as Photofit (e.g. Cutler, Stocklein and Penrod, 1988; Wogalter and Marwitz, 1991; Kovera, Penrod, Pappas and Thill, 1997; Koehn and Fisher, 1997; Davies, van der Willik and Morrison, 2000; Brace, Pike and Kemp, 2000) and newer systems can produce very good likenesses under some circumstances (e.g. Frowd et al., 2005; Frowd et al., 2005; Frowd et al., 2010). However, despite these improvements, facial composites often still portray a very poor resemblance to the suspect/target. This is because constructing a facial composite is an incredibly difficult task. A witness is asked to remember the face of someone that they will have only seen once, perhaps for just a very short period of time. The image is then displayed in the media and in police stations in the hope that someone who is familiar with the suspect will recognise them from the composite. As composites are important investigative tools it is vital that researchers examine methods that may be able to assist a witness and ultimately improve suspect/targetresemblance in the facial composite.

Much of this research has tended to concentrate on developing methods of improving the likeness of facial composites after they have been constructed. For example, researchers have examined morphing composites from single and multiple witnesses (e.g. Brace et al., 2006; Bruce et al., 2002), caricaturing (Frowd et al., 2007) and by manipulating expression (Mcintyre et al., unpublished). Although this research has had some success in improving recognition rates, no research to date has examined the impact of viewpoint on both the construction of a facial composite and the subsequent identification of the image. Viewpoint in this context refers to the relative horizontal rotation of the target face to the viewer (e.g. full-face, three-quarter and profile views).

Consideration of viewpoint is important because in a real-life situation a witness will have viewed a previously unfamiliar three-dimensional moving face. However, when a witness is invited to build a composite likeness of the face, they are asked to construct a two-dimensional full-face image. Evidence on the role of movement in unfamiliar face recognition has indicated that movement may help to build a robust three-dimensional representation of the face (e.g. Schiff, Banka and De Bordes Galdi, 1986; Bruce and Valentine, 1988; Pike, Kemp, Towell and Philips, 1997). As this research suggests that a witness may have encoded and stored a three-dimensional representation of the face, this raises the issue of whether a full-face composite is the best viewpoint to use. Would it be easier for a witness to construct a three-quarter view composite, rather than a full-face composite?

Furthermore, a witness may have only seen a side view of the face. When this is the case, is it appropriate to ask them to construct a facial composite in a different view? It also unclear whether constructing a composite in a single view sufficiently captures the three-dimensional nature of the face to facilitate later identification of the 
composite image. While no research to date has examined these issues in facial composite construction, researchers have investigated whether one particular view is preferred in face recognition (e.g. Bruce, Valentine and Baddeley, 1987; Schyns and Bülthoff, 1994; Hill, Schyns and Akamatsu, 1997; Newell, Chorizo and Valentine, 1999; Lui and Chauduri, 2002). This research stems partly from research on object recognition that has suggested not only that object recognition may be viewpoint dependent (e.g. Edelman and Bülthoff, 1992; Tarr and Pinker, 1990), but also that certain views of an object are often preferred (e.g. Palmer et al., 1981).

For recognition of faces, it was speculated that as a three-quarter view is centred between the full-face and profile views, it may contain information that is available in both views. As such, a three-quarter view could represent a canonical view in face recognition. When multiple views of a face were presented at study, some researchers have reported that profile views $\left(90^{\circ}\right)$ performed poorly but recognition performance for full-face $\left(0^{\circ}\right)$ and three-quarter views $\left(45^{\circ}\right)$ did not differ significantly (Hill, Schyns and Akamatsu, 1997; Newell, Chorizo and Valentine, 1999, Experiment 3). Similar results were obtained by Logie, Baddeley and Woodhead (1987, Experiment 4) using 'live' targets. However, Bruce, Valentine and Baddeley (1987) did find a three-quarter view advantage but only for unfamiliar faces, not familiar ones. Baddeley and Woodhead, (1983) and Krouse (1981) also reported a similar threequarter view advantage for previously unfamiliar faces.

This suggests that there may be a three-quarter-view advantage when more than one view is presented at study. However, Liu and Chaudhuri (2002) and others (e.g. Laughery, Alexander and Lane, (1971, Experiment 2; Davies, Ellis and Shepherd (1978, Experiment 2) have also failed to find such an advantage. This led Liu and Chaudhuri (2002) to suggest that there is little evidence for a three-quarter-view advantage. Indeed, Schyns and Bülthoff (1994, Experiment 1) compared two different side views $\left(18^{\circ}, 36^{\circ}\right)$ with a full-face view and found that no one view was preferred. It seems therefore that when multiple views have been encoded at study, recognition performance is equivalent for both the full-face and three-quarter view.

When single full-face images have been presented at study, researchers have failed to find a three-quarter view advantage at the test phase (Newell, Chorizo and Valentine, 1999, Experiment 1; Patterson and Baddeley, 1977; Woodhead, Baddeley and Simmonds, 1979). However, the three-quarter view does seem to have an advantage over the full-face view in terms of generalising to novel views. One reason for this may be the symmetry hypothesis (Schyns and Bülthoff, 1994; Troje and Bülthoff, 1996; Hill, Schyns and Akamatsu, 1997; Troje, 1998) which might also help to explain some of the mixed findings in the literature. This hypothesis suggests that as a face is essentially bilaterally symmetrical (albeit not perfectly), side-views of a face can be thought of as non-singular, as a symmetrical view can be generated from them, whereas full-face views are singular, as a symmetrical view cannot be generated. The symmetry argument suggests that when a three-quarter view is presented at study, a 'virtual view' could be generated and this may result in the successful recognition of the face in a novel view i.e. the full-face. Schyns and Bülthoff (1994, Experiment 2) examined this and found a strong generalisation effect for $36^{\circ}$ and $-36^{\circ}$ faces, compared to $18^{\circ}$ and $0^{\circ}$. The authors also reported an inverted $U$ shape performance for the full-face $\left(0^{\circ}\right)$ for recognition accuracy with sharp decreases in performance for each increased angle of rotation. Similar results were obtained by Hill, Schyns and 
Akamatsu (1997, Experiment 2) using full-face, three-quarter view (45 $)$ and profile $\left(90^{\circ}\right)$ views. A peak in performance was observed for the opposite three-quarter view - the symmetrical view. Furthermore, Hill, Schyns and Akamatsu (1997, Experiment 3) report that while the full-face and three-quarter views did not generalise well, generalisation from a three-quarter view did not depend on the test view.

These results all suggest that unfamiliar face recognition is viewpoint dependent and that generalisation to novel views from only one view is dependent on the learning view. More importantly, different patterns of viewpoint dependence are observed for different learned views. In particular, performance for the full-face view appears to reflect an inverted U shape function. Similarly, while generalisation performance for side views also decreases slightly, there is often a peak in performance for the opposite view - the symmetrical view. This suggests that learning a side view of a face may result in better generalisation performance than learning a full-face view.

So, it is clear that unfamiliar face recognition is viewpoint dependent. However, it is unclear whether the same pattern of results would be observed in a facial composite task, which, as a reconstruction task involves more than simply recognising a face. In particular, with systems such as PROfit composite construction can be described as primarily a recall task. When new features are presented, witnesses search their memory, extract information and decide whether the presented feature 'matches' the feature stored in memory. The process of recalling a face/feature, is inherently more difficult than recognising a face, which may be facilitated by familiarity rather than conscious recollection. Indeed, authors have noted the reconstructive nature of recall (e.g. Bartlett, 1932; Davies, Ellis and Shepherd, 1978). In particular, Davies et al., (pg. 22) state that "Photofit making...becomes an act not of reproduction but of reconstruction...". Furthermore, Bartlett (1932) argued that stored items could become combined at retrieval, resulting in the recollection of incorrect information, thus suggesting that successful retrieval may be dependent on the cues available at test. Indeed, the encoding specificity principle (e.g. Tulving and Thomson, 1973) states that retrieval will be more successful when retrieval cues more accurately match those in the original encoded experience. In composite construction, the retrieval cues (i.e. the facial features) in a composite will never precisely match those in the original suspect/target face. However, retrieval may be more successful when the cues (features) are displayed in a more three-dimensional way i.e. in a three-quarter-view, rather than in a full-face view which captures very little of the three-dimensional information that would have been available at encoding. Furthermore, retrieval may be further enhanced when viewpoint is matched at both encoding and retrieval (construction).

\section{Aims}

The present study set out to investigate the importance of viewpoint in facial composite construction by examining whether participants could construct a more identifiable composite in a three-quarter view, compared to the standard full-face view. In the first experiment participants were asked to construct both a full-face composite and a three-quarter-view composite after they had been presented with all views of a face. Presenting all views should ensure that any difference between these two sets of composites would be due to the view during construction and not at encoding. All target faces were presented on video and displayed equal amounts of all views, in an attempt to emulate everyday interaction. The second aim was to 
investigate the issue of encoding specificity (e.g. Tulving and Thomson, 1973) and viewpoint dependency in more detail and this experiment also investigated whether composites would increase when the retrieval cues (features) were more similar to encoding (i.e. more three-dimensional).

As well as investigating ways to improve the construction of composites, experiment 2 also examined whether the use of different views could improve identification rates for composites after they had been constructed. Recent research has found that presenting varied information from multiple witnesses does increase identification rates. In particular, showing multiple composites (Brace et al., 2006) or combining composites from four different witnesses increased the number of correct identifications above the level observed for a single composite (Bruce et al., 2002). Similarly, combining composites from the same witness using two different composite systems also increased identification significantly (Ness et al., 2003). This benefit appears to be primarily driven by the presentation and combination of varied information from different witnesses. As two different views of the same person can look very different and may contain different kinds of information (more 3D structure in the three-quarter-view composite) this investigation examined whether presenting both full-face and three-quarter view composites together would increase identification above the level observed for a single composite.

At present, although there are no guidelines (e.g. ACPO, the Association of Chief Police Officers and National Policing Improvement Agency, 2009) prohibiting the construction of more than one composite by a witness, it is implied in the guidelines that "Each witness provides an individual image separately from all other witnesses" (section 4.3a). Therefore, as well as asking participants to construct composites in both views (full-face and three-quarter), three-quarter-view composites were also automatically generated from the full-face composites using PROfit. From a theoretical perspective, this would further enhance our understanding of any viewpoint effects. If the three-quarter-view acts as a more efficient retrieval cue then the constructed composites should contain a more accurate likeness of the target than the automatically generated ones. From an applied perspective, if performance increases when the full-face and automatically generated composites are presented together, then the use of an additional generated composite could be beneficial as it would mean a witness would still only need to construct a single composite, as per existing guidelines.

Experiment 2 examined whether the presentation of both three-quarter and full-face view composites would increase performance above the level achieved for a single composite.

Experiment 3 examined the encoding specificity principle in more detail. View at study was a between-subjects factor (full-face, three-quarter and all views) while construction view (full-face and three-quarter-view) was a within-subjects factor. That is, participants were allocated to one viewing condition and were required to construct two composites of the target (one in a full-face view and one in a three-quarter-view). At test, full-face and three-quarter-view composites were presented alone and in pairs (i.e. one full-face and one three-quarter-view that had been constructed by the same participant). 
In this investigation all composites were constructed using the PROfit composite program. The standard construction procedure is fully described in Fodarella, Kuivaniemi-Smith and Frowd (2015). For this particular investigation the two female databases were used (full-face and three-quarter view ${ }^{1}$ ). These both contained 343 hairstyles, 281 faces shapes, 214 eyes, 316 noses, 317 lips, 76 eyebrows and 51 ears. In order to create the databases two photographs were taken of each volunteer - one at full-face and one at three-quarter view. Four features were then taken from each of these photographs. In order to create the 'generation' procedure, every feature was given 'anchor points' in order to determine its location within the face and a threedigit identity code. When the program is asked to generate a composite, it uses an index table to correctly identify the full-face features and the corresponding threequarter view features (ensuring that matching features are used).

\section{Experiment 1}

This experiment used targets that were female members of staff from the psychology department at the University of Stirling. In the first stage of the experiment unfamiliar participants viewed a video of a female target. They were then asked to construct two composites of her face from memory (one at full-face and one at three-quarter-view). In stage 2 a further set of three-quarter-view composites were automatically generated from the full-face composites. In stage 3, participants who were unfamiliar with the targets rated the composites for likeness. In stage 4, participants who were familiar with the targets attempted to identify the composites.

\section{Stage 1: Construction of Composites Materials}

In this experiment target faces were taken from the same video and photographic database used to create the female database in PROfit. It was not possible to construct an image that was an exact match for a target face (i.e. contained all of the target face's features), as only a maximum of five features were taken from each photograph. This is obviously not a particularly ecologically valid situation, as in reality none of the suspect's features would be in the database used by a composite system. However, this procedure permitted an initial examination of the relationship between featural information and composite performance by, for example, allowing the measurement of 'likeness' to the target through the number of features from the target that appeared in the composite image.

In stage 1, the target faces were initially shown to the participants in 30 second video clips. These clips comprised video frames from the female database that were extracted and digitised without sound, using the Media 100 video-editing package. A thirty-second video clip was captured for each target. Each clip consisted of fifteen seconds of movement (rotating in chair from left to right: shaking head from side to side, nodding up and down) and fifteen seconds of full-face view.

\footnotetext{
${ }^{1}$ This database displays composites at a $30^{\circ}$ angle, which is consistent with research
} (e.g. Troje and Bülthoff, 1996) which has found optimal performance for recognition between $25^{\circ}$ and $40^{\circ}$ 
Composites were constructed using PROfit (Windows version 3.0) on an ASUS HiGrade UltiNote AS8400 laptop computer.

\section{Participants}

Sixteen adults aged between eighteen and forty years and the same ethnicity as the targets, were recruited from the psychology department of Queen Margaret University College, Edinburgh. All participants were unfamiliar with the targets. Each participant received a $£ 10$ payment.

\section{Design}

A 4 (target) by 2 (construction view) mixed design was adopted, with target as a between-subjects factor and construction view (full-face and three-quarter view) as a within-subjects factor. Each participant viewed a thirty-second video clip of one target and constructed two composites of the same target (one in a full-face view and one in a three-quarter view) from memory. There were four targets and sixteen participants, creating a total of thirty-two composites ( 8 per target). The order of construction was counterbalanced so that eight subjects constructed a three-quarter view composite first and eight constructed a full-face composite first. The result was that sixteen full-face (4 per target) and sixteen three-quarter-view composites (4 per target) were constructed.

The sixteen full-face composites were then used to generate a further set of threequarter view composites using PROfit. This created another sixteen composites and resulted in a total of forty-eight composites (16 full-face, 16 three-quarter-view created by a participant and 16 three-quarter-view generated automatically). See Figure 1 for an example and Stage 2 for a description of the procedure.

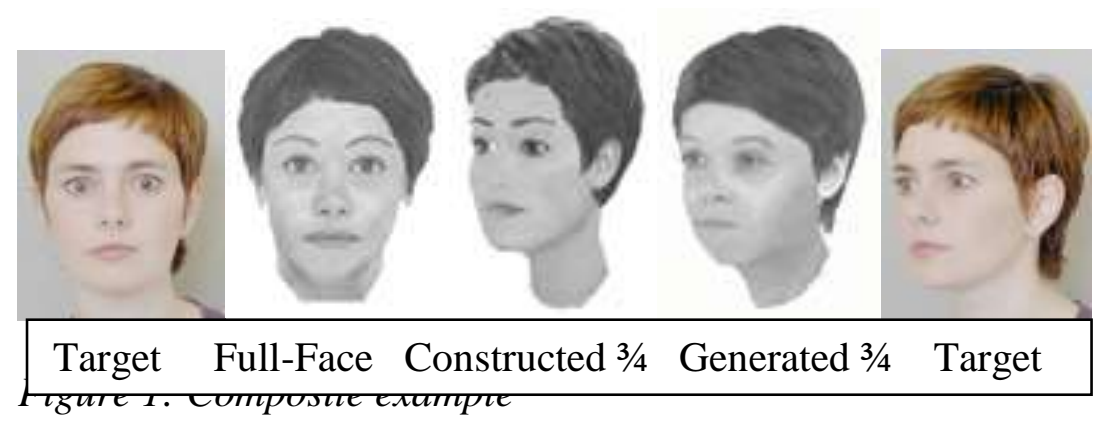

\section{Procedure}

Stage 1: Each participant was asked to view a thirty-second video clip. The participant was not initially told that they would have to remember this person. After the participant had viewed the clip they were informed of the true nature of the experiment. The procedure for the cognitive interview (Memon, Wark and Fraser,2010) ('elements of which were used to elicit recall of the face) and construction of the composites was then explained. As rapport building is an important aspect of the cognitive interview procedure (prior to eliciting a description), the experimenter then chatted to the participant about their interest/work etc in order for them to feel as relaxed and familiar with their surroundings as possible. The total average (mean) time spent on explanations and rapport building was 12 minutes. The participant was then encouraged to close their eyes and visualise the face. For the first recall attempt (free recall) they were asked to describe the features in any order and were encouraged to describe everything they could see, even if they thought it was 
irrelevant. The second recall attempt was more structured in that the participant was asked to focus on each feature separately, starting at the top of the head and working their way down the face slowly. If a third recall attempt was needed the order was varied (e.g. starting at the bottom of the face and working upwards). If the participant had omitted any information, questions were then directed at these areas (e.g. Can you recall/describe the shape of the mouth?). No questions were directed at features or aspects of features that the participant had said that they could not recall. This description was then entered into either the full-face or three-quarter view database in PROfit.

PROfit is very similar to other computerised composite systems as it displays a small facial shaped icon. A drop-down menu that provides a breakdown of each part of the feature accompanies every feature in this icon. For example, when you click on the face, the drop-down menu displays 'face shape, chin shape, length, width, age, fleshiness, forehead' etc. Within each of these categories there are a range of options. For example, for 'face shape' the options are 'oval, round, triangular, square and angular'. If a descriptor did not match the word(s) the participant had used to describe that feature, then the participant chose the descriptor that they felt was the closest alternative. The experimenter offered no advice. If a participant did not recall a feature or aspect of a feature e.g. size of eyes, then the 'average' option was entered. Where this was not possible, no descriptor was chosen.

When the description had been entered into PROfit, the participant and experimenter worked together to produce a facial likeness, by viewing chosen features, selecting alternative features and editing both features (e.g. changing size, shape, shade etc) and configuration. All features were edited using the tools available in PROfit. If further alterations were needed (e.g. highlights, shadows, laughter lines) the composite was exported into Adobe Photoshop 7. Construction of the composite ceased when the participant was either confident that the image represented a good likeness of the target, or indicated they could not make any further changes.

On completion of the first composite, the description that was elicited from the interview was then used to construct the second composite (i.e. the same description that was used to construct the first composite). The description was entered into the second PROfit database (either full-face or three-quarter view). Both databases contain the same features but they are not in the same order, so this helps to ensure that the participant cannot just remember the number or the order of the features and choose the same one, thereby replicating the first composite in a different view. The participant and experimenter then worked together to construct the second composite. No suggestions were offered during construction of this second image. No time limit was placed on the construction of either composite. The total average time to conduct the cognitive interview and construct both composites was 90 minutes.

\section{Stage 2: Automatic generation of three-quarter view composites}

A further set of three-quarter view composites were automatically generated from the full-face composites. In order to generate the image PROfit uses an index table to ensure that matching features are used. However, any alterations that are made to the full-face composite by the witness are not 'transferred' to the automatically generated image. As a result, a detailed list of all alterations was kept by the operator and each generated composite was then altered in exactly the same way as the original full-face 
composite, for example if the fringe had been removed on the full-face image it was removed on the generated image. This procedure commenced when all of the composites had been constructed and was repeated for all sixteen full-face composites. The participants were not present during this process.

\section{Stage 3: Evaluation of Composites \\ Likeness Ratings \\ Materials}

Each full-face and three-quarter view composite was presented with monochrome photographs depicting the target in both views (one in full-face and one in threequarter view). This ensured that as much information as possible was available for the task. All images measured $13 \mathrm{~cm}$ in height. The photographs were edited using Microsoft Photo Editor to ensure that brightness and contrast were constant.

\section{Participants}

Forty unpaid participants aged between 18 and 57 years were recruited from Queen Margaret University College and local Tesco supermarkets. All were the same ethnicity as the target faces and they were also unfamiliar with the targets.

\section{Design}

Unfamiliar participants rated the composites for likeness on a scale from one (low) to ten (high). The composites were divided into two books each containing twenty-four composites ( 8 full-face, 8 constructed $3 / 4$ view and 8 generated composites with an equal number for each of the targets). Each participant saw only one book, with twenty participants rating the composites in book one and twenty rating the composites in book two. Each composite was printed on a single sheet of A4 paper and displayed with two monochrome photographs of the target (one full-face and one three-quarter view, printed side by side on a separate sheet of A4 paper). Presentation order was randomised.

\section{Procedure}

Each participant was told that the composites were constructed after a 'participant witness' had only seen the target face for 30 seconds. It was stressed that the composites were constructed from memory and that they represented a likeness of the original target. Each participant was then informed that his or her task was to rate how good the likenesses were. They were asked to study each set of images (composite and photographs) and rate the composites for likeness on a scale of 1 (low) to 10 (high). This was repeated for all twenty-four composites. No time limit was placed on this procedure.

\section{Results}




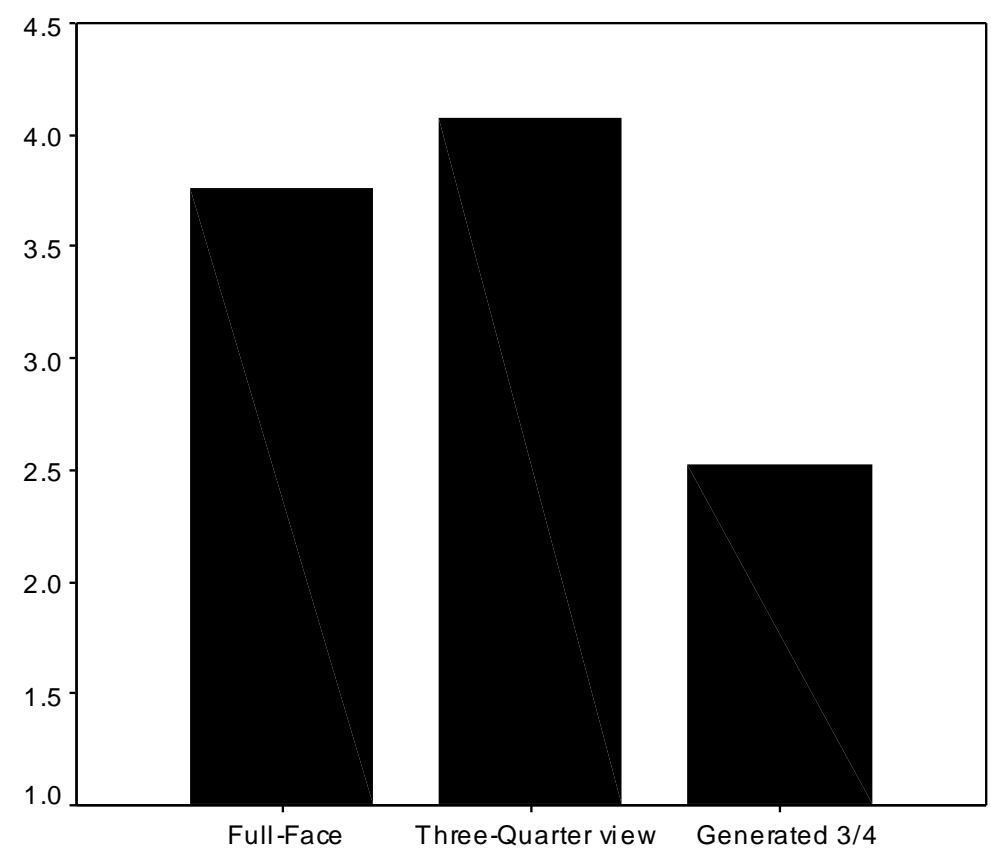

Figure 2: Mean ratings for each type of composite

From figure 2 it can be seen that the highest likeness ratings were achieved for the three-quarter view composites $(M=4.1, S D=0.95)$ followed by the full-face composites $(M=3.7 S D=1.27)$ then the generated three-quarter view composites $(M=$ 2.5, $S D=0.89$ ). A 3 (composite type) by 4 (target) analysis of variance was conducted which revealed a significant main effect of composite type $[F(2,78)=32.031, p<0.001$, $\left.\bigcap \mathrm{p}^{2} .451\right]$. Further analysis revealed that the generated three-quarter view composites were rated significantly lower similarity than both the full-face composites $[\mathrm{t}(39)=$ $6.555, \mathrm{p}<0.01]$ and the three-quarter view composites that had been constructed by the participant-witnesses[ $(\mathrm{t}(39)=8.058, \mathrm{p}<0.01]$. The analysis also revealed a significant main effect of target $\left[\mathrm{F}(3,117)=26.146, \mathrm{p}<0.001, \eta \mathrm{p}^{2} .401\right]$ and a significant interaction between composite type and target $\left[\mathrm{F}(6,234)=7.348, \mathrm{P}<0.001, \eta \mathrm{p}^{2} .159\right]$. Further analysis revealed that for three of the targets the full-face and three-quarter view composites were rated significantly higher than the automatically generated threequarter view composites. One target, target 2 , was rated poorly across all three conditions.

More specifically, for target 1 , the three-quarter view composites $(\mathrm{M}=5.18, S D=1.1)$ were rated as better likenesses than both the full-face $(M=3.87, S D=1.7)$ and generated three-quarter view composites $(\mathrm{M}=2.36, S D=1.50,(\mathrm{p}<0.001$ for both respectively). The ratings for target 2 were poor across all three conditions and did not differ significantly [p,0.05] (Full-face, $M=2.65, S D=1.32$; three-quarter view, $M=$ 2.51, $S D=1.17$; generated three-quarter view, $M=2.4, S D=1.5$ ). For target 3 , the three-quarter view composites $(M=4.1, S D=1.6)$ were rated as better likenesses than the full-face composites $(M=, 3.5, S D=1.9)$ and the generated composites $(\mathrm{M}=2.4$, $S D=1.3$ ). However, there were no significant differences between the full-face and three-quarter view composites but both were rated significantly higher than the generated three-quarter view composites $[\mathrm{p}<0.05]$. A similar pattern was observed for target 4 . There were no significant differences between the full-face and three-quarter view composites (Full face, $\mathrm{M}=4.3, S D=2.1$; three-quarter view, $M=4.5, S D=1.8$ ) but 
both were rated significantly higher than the generated three-quarter view composites $(M=2.8, S D=1.5)$.

As the composites were split into two different ratings booklets, a composite type (3) by ratings booklet (2) ANOVA was conducted which again revealed a significant main effect of composite type but no main effect of ratings booklet $[\mathrm{F}(1,39)=2.780$, $\left.\mathrm{p}>0.05,1 \mathrm{p}^{2} .067\right]$ and no interaction $\left[\mathrm{F}(2,78)=1.595, \mathrm{p}>0.05,1 \mathrm{p}^{2} .151\right]$.

As each participant constructed two composites, further analysis was conducted on the order of construction. This revealed no effect of order of construction $[\mathrm{F}(1,15)=$ $.712, \mathrm{p}>0.05]$.

\begin{tabular}{|l|l|l|l|}
\hline & Full-face & Three-quarter view \\
\cline { 2 - 4 } & Target 1 & 2 & 3 \\
\cline { 2 - 4 } & Target 2 & 0 & 1 \\
\hline Target 3 & 3 & 1 \\
\hline Target 4 & 1 & 2 \\
\hline Total & $\underline{6}$ & $\underline{\mathbf{6}}$ \\
\hline
\end{tabular}

Table 1: Frequency of correct feature choices

Table 1 shows the number of times a correct feature was chosen during the composite construction process, broken down by type of composite and target. As can be seen, the frequencies are low and do not differ between the different views. The individual features that were correctly chosen by different participants were ears (2), nose (2), eyes (3), hair (6). No single participant chose more than one correct feature. This is comparable to previous research (Koehn and Fisher, 1997) who also reported very few correct features using the Mac-A-Mug Pro system. In their investigation twentyfive composites did not have any correct features, 19 composites had one correct feature and two composites had two correct features.

\section{Stage 4: Identification}

\section{Participants}

Thirty-two members of staff from the department of psychology at the University of Stirling participated in Stage 4. All participants were familiar with the target faces and were aged between 23 and 58 years old.

\section{Design}

Participants who were familiar with the targets were asked to identify the composites. If participants were presented with more than one composite of the same target then their identification responses may have been influenced by that prior exposure. I.E. they may be more likely to identify a target after seeing three composite images of them. In order to limit these priming effects, each participant was presented with only one composite image for each target. Twelve books were constructed, each containing one type of composite for each of the four targets. Each participant saw only one book (i.e. four composites) which contained one composite of each target and at least one of each type of composite (full-face, three-quarter view and generated three-quarter view).. 


\section{Procedure}

Each participant was informed that the composites were constructed after a 'participant witness' had only seen the target face for 30 seconds. It was stressed that the composites were constructed from memory and that they represented a likeness of the original target. Subsequently each participant was then informed that the target was a familiar person and that his or her task was to try to identify that person from the composite. The first three participants assumed that the targets were of famous rather than personally familiar faces. This was not because the composites looked like famous targets (these participants could not identify the composites as famous targets), but rather that many members of staff regularly take part in experiments where famous faces are used. Therefore it was necessary to eliminate these three participants from the study and to change the task instructions. Each participant was then told that the composite represented someone from the psychology department. While these instructions decreased the number of possible targets (to members of the department), the total number of targets were still 32. Participants were encouraged to provide a name or some identifiable semantic information about the person. On completion, participants were told who the targets were.

\section{Results}

The percentage of correct identifications and false positives was similar for both the full-face composites ( $23 \%$ correct with $9 \%$ false positives), and the three-quarter view composites $(22 \%$ correctly identified with $9 \%$ false positives), with the generated composites performing more poorly (13\% correctly identified and $28 \%$ false positives). A identification was counted as correct if the participant produced either the correct name of the target, or provided specific identifiable semantic information about the person. If a participant provided an incorrect name or provided semantic information that identified another person, this was counted as a false positive.

The data was collapsed across targets and a Friedman test was conducted on the hit rate. This revealed that there were no significant differences $\left[\mathrm{X}^{2}(2)=1.55, \mathrm{p}>0.05\right)$ between the different types of composite although the trend is clearly in line with the rating scores. These identification rates are low but they are not unusual for facial composites and are in line with rates observed in previous research.

\section{Discussion}

The results from this experiment did not show a three-quarter view advantage, but instead revealed that the three-quarter view composites performed as well as the fullface composites. This finding is in line with face recognition research (e.g. Hill, Schyns and Akamatsu, 1997; Schyns and Bülthoff, 1994) which has found that when all views were presented at study, no one view is preferred at test. As a result of exposure to all views, sufficient information may have been encoded and resulted in successful generalisation to either of the two views. The generated three-quarter view composites performed poorly and while it may be advantageous practically to automatically generate an additional composite, it is clear that generating an image from a full-face composite does not result in a good target likeness. The issue of encoding specificity (e.g. Tulving and Thomson, 1973) will be explored later in experiment 3 where viewpoint at encoding and construction is investigated in more detail. 


\section{Experiment 2}

Experiment 1 examined whether constructing an image in a three-quarter-view would produce more identifiable composites. The results suggested that there was not a three-quarter view advantage and instead revealed that the three-quarter view composites were as good as the full-face composites. The aim of experiment 2 was to investigate this further by examining whether presenting both composites (full-face and three-quarter view) would increase identification rates above the level observed for a single full-face composite. While likeness ratings and identification rates were similar for both types of composites, it is possible that the three quarter view composites may contain either different types of information or more information than the full-face composites (e.g. more 3-dimensional information about the structure of the face). As two different views of the same face can look very different and often more different than two different people, presenting both composites together may serve to increase identification rates in a manner that has been observed in previous research using composites from multiple witnesses and systems (e.g. Brace et al., 2006; Bruce et al., 2002; Ness et al., 2003). Stage 1 examines this for composites that have been constructed by the same participant-witness. Stage 2 repeats stage 1 but with the automatically generated three-quarter view composites. This experiment used the composites that had been constructed and generated in experiment 1 .

\section{Stage 1:Presenting full-face and three-quarter view composites Participants}

Thirty-two participants were recruited from the psychology department at the University of Stirling. They consisted of third and fourth year psychology students and three members of staff. All participants were familiar with the targets. They ranged in age from 21 to 53 years.

\section{Design}

As the aim of this experiment was to examine whether adding an additional image (a three-quarter-view) would increase identification rates, this experiment used the composites that had been constructed in experiment 1 . In order to identify which composites to use, the likeness ratings data from experiment 1 was examined. From this it was possible to identify the full-face composites that were rated very poorly, those that were rated as 'average' (intermediate) and those that were given the highest ratings. The poor composites were not used in this experiment as it is unlikely that adding another poor composite would increase identification. Therefore the intermediately and highest rated composites were chosen. See table 2 for the mean ratings for the full-face composites and their corresponding three-quarter view composites (i.e. the ones constructed by the same participant).

\begin{tabular}{|l|l|l|}
\hline Target & Full-face & 3/4 view \\
\hline 1 Inter & 3.45 & 3.6 \\
1 Best & 6.25 & 5.65 \\
\hline 2 Inter & 4 & $\mathbf{7 . 0 5}$ \\
2 Best & 6.25 & 3.3 \\
\hline 3 Inter & 2.9 & 1.55 \\
3 Best & 5.1 & $\mathbf{6 . 0 5}$ \\
\hline 4 Inter & 3.85 & $\mathbf{4 . 1}$ \\
4 Best & 5.8 & 3.35 \\
\hline
\end{tabular}




\section{Table 2: Mean ratings for the intermediate and highest rated composites used in this experiment}

As illustrated in table 2, only three of the three-quarter view composites were rated higher than the corresponding full-face images, making it unlikely that any potential advantage of presenting both views would be a result of simply adding in a better composite.

As the aim to examine whether presenting both composites would increase identification above the level observed for a single composite, the full-face composites were presented alone and then with their corresponding three-quarter view (i.e. the one that was constructed by the same participant). This created a total of sixteen presentations ( 4 best and 4 intermediate at full-face alone and 4 best and 4 intermediate at full-face and three-quarter view together). Participants were only shown one type of composite for each of the four targets. This resulted in each participant viewing 1 best full-face alone, 1 best full-face and three-quarter view, 1 intermediate full-face and 1 intermediate full-face and three-quarter view for each of the four targets.

\section{Procedure}

Participants were approached and asked to attempt to identify the composites. No participant had taken part in Experiment 1. The procedure was identical to the original (revised) identification procedure in stage 3 of Experiment 1.

\section{Results and discussion}

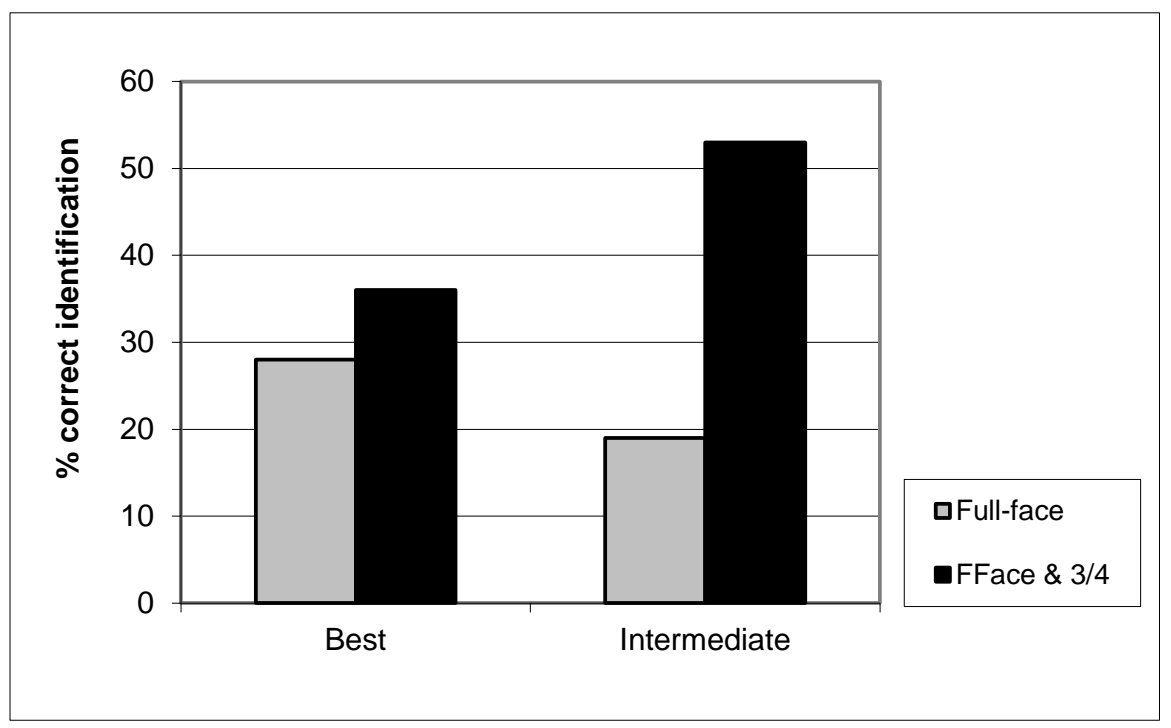

\section{Figure 3: (\%) correct identifications for the full-face and full-face with three- quarter view presentations}

Figure 3 illustrates the percentage correct identifications for presenting the single fullface composites alone and with their corresponding three-quarter view. The data was collapsed across target and a Cochran's Q test revealed that the full-face and threequarter composites shown together were identified significantly more accurately than the single full-face composites $[\mathrm{Q}(3)=8.43, \mathrm{p}<0.05)$. Further analysis using $\mathrm{McNemar}$ tests revealed that there were significant differences between the intermediate rated 
full-face and full-face \& three-quarter composites [p<0.05], but not between the best composites [ $p>0.05]$. In particular, the intermediate composites displayed a marked increase jumping from $19 \%$ correct identifications for the single full-face to $53 \%$ when both views were presented. The best composites increased from $28 \%$ for the single full-face to $37 \%$ when both views were presented.

This result is particularly interesting given that the intermediate full-face composite for target 2 was the only intermediate composite that was presented with a threequarter-view that was of much better quality (see table 2). An examination of the intermediate composites by target revealed significant increases for presenting both views for targets 1 and 4 [p < 0.05] but not for targets 2 and 3 [p $>0.05]$. With such a small pool of targets it is invariable that some differences will emerge, however it is unclear at present why a significant increase was observed for the intermediate composites and not for the best composites. In general however, these results are consistent with previous research (e.g. Brace et al., 2006; Bruce et al., 2002) by indicating that presenting more information improves the identification of composites. They are also important from an applied perspective. As there is no way of knowing whether a composite is 'average' or 'good', the results from this experiment suggest that asking a witness to construct two composites of the same person, in different views would be advantageous regardless of quality.

\section{Stage 2: Presenting full-face and 'automatically generated' three-quarter views}

In experiment 1 the automatically generated composites performed poorly when presented alone, in comparison to the constructed composites. However, they may still facilitate identification when presented with their corresponding full-face composite. In order to examine this, the same full face composites that were used in Stage 1 of experiment 2 were used here. However, instead of presenting them with their constructed three-quarter view composites, they were also presented with their corresponding automatically generated three-quarter view.

\section{Materials}

An identification task was not undertaken due to the limited number of participants who were familiar with the targets. Instead, a six alternative forced-choice task was undertaken. To create the arrays, five distractors were chosen for each of the four target faces and they were matched for hair style/colour, face shape and age. These were presented with the target as black and white photographs on a single sheet of A4 paper. Microsoft Photo Editor was used to ensure that brightness and contrast was consistent.

\section{Participants}

Forty-eight participants aged between 17 and 50 years were recruited from local businesses in Edinburgh. All participants were unfamiliar with the targets. No participant had taken part in any of the previous experiments.

\section{Design}

The same best and intermediately rated composites were used. The full-face composites were shown alone and both with their corresponding three-quarter view and generated composites. There were twenty-four composite types in total ( 8 fullface, 8 full-face and constructed three-quarter and 8 full-face and automatically generated three-quarter). As in Stage 1 of experiment 2, each participant was shown one type of composite for each of the four targets. Each composite type was presented 
with an array of six black and white photographs (one of the target and five distractors). This method was used as a way of assessing the quality of the composites and was not designed as a formal 'line-up'.

\section{Results}

The overall percentage correct matches were $52 \%$ for the single full-face composites, $73 \%$ for the full-face with constructed three-quarter view composites and $42 \%$ for the full-face with generated composites. A Friedman test revealed that the observed differences were significant, $\left[\mathrm{X}^{2}(2,48)=7.078, \mathrm{p}<0.05\right]$. Further analyses using Wilcoxon Signed Rank tests revealed that there were significantly more correct matches for the full face with the constructed three-quarter view composites, compared to the full-face and automatically generated composites $[p<0.05]$. The difference between the full-face with three-quarter view and the single full-face composites did not quite reach significance $[\mathrm{p}=0.068]$, although the trend is clearly in line with the results obtained in experiment 1 . In addition, there were no significant differences between the single full-face and the full-face and generated composites $[\mathrm{p}=0.369]$.

\section{Discussion}

These results suggest that while there appears to be a benefit for presenting two views, this benefit is only apparent when the composites have actually been constructed. The automatically generated composites performed poorly when presented alone (experiment 1) and when presented with their corresponding full-face composite. These results suggest that a three-quarter-view composite does act as an efficient retrieval cue, as performance is significantly better for the constructed threequarter composites compared to the automatically generated images. The results also suggest that just presenting more information does not facilitate increased performance at test. Instead, these results provide supporting evidence for the presentation of different types of information, as reported by Brace et al., (2006) and Bruce et al., (2002)

The results from Experiment 1 revealed that a three-quarter-view performed as well as a full-face view when all views were presented at study. This is line with face recognition research (e.g. Hill, Schyns and Akamatsu, 1997; Schyns and Bülthoff, 1994). The next experiment examined the effect of encoding specificity (e.g. Tulving and Thomson, 1973) in more detail. Participants were allocated to one of three encoding conditions (full-face, three-quarter-view or all views). They were then asked to construct both a full-face and a three-quarter-view composite of the same target.

\section{Experiment 3}

Experiment 1 examined whether participants could construct a more identifiable composite in a three-quarter view, compared to the standard full-face view. Participants were asked to construct both a full-face composite and a three-quarterview composite after they had been presented with all views of a face. The results from this experiment found that when participants constructed a composite in a threequarter view, performance was as good as a full-face view but not better. Furthermore, it was found that automatically generating a three-quarter view composite from a full-face composite resulted in a poor likeness. Experiment 2 examined whether adding an additional three-quarter view composite to a full-face composite would increase identification. For the constructed composites identification 
increased markedly for the intermediately rated full-face composites. No benefit was observed for presenting a full-face composite with an automatically generated threequarter view composite.

The aim of the third experiment was to investigate the issue of encoding specificity and viewpoint dependency in more detail. In a real-life situation a witness may have only seen one view of a face. Therefore, the view at both encoding (full-face, $3 / 4$, all views) and test (full-face, $3 / 4$ ) was manipulated. If the encoding specificity principle (e.g. Tulving and Thomson, 1973) is correct, greater performance should be observed when the encoding and construction views match.

\section{Stage 1: Construction of Composites Materials}

Four females from a different university (Queen Margaret University College, Edinburgh) agreed to act as targets in this experiment. Each target was videotaped individually using a Sony Hi8 camcorder for approximately three minutes. They were asked to sit in a chair and converse with an experimenter while both looking straight ahead and moving (rotating in chair from left to right: shaking head from side to side, nodding up and down). Three thirty-second video clips were then created for each target. The first clip displayed the target looking straight-ahead (full-face condition), the second clip displayed the target at a thirty degree angle (three-quarter view condition) and the third clip displayed equal amounts of the previous two conditions (15 seconds looking straight ahead and 15 seconds of movement: the all view condition). Frames were extracted and digitised without sound, using the Media 100 video-editing package. Targets were also photographed using a Digital Olympus C900 camera in two different positions (full-face and three-quarter view).

\section{Participants}

Twenty-four adults aged between eighteen and forty years were recruited from Stirling University. All participants were unfamiliar with the targets. Each participant received a $£ 10$ payment.

\section{Design}

A 4 (target) by 3 (encoding view; full face, $3 / 4$ view or all views) by 2 (construction view; full face or $3 / 4$ view) mixed factorial design was adopted. Target and encoding view (full-face, three-quarter view and all views) was a between-subject factor and construction view (full-face and three-quarter view) was a within-subjects factor. As such, each participant saw one unfamilar target in one viewing condition (either fullface view, three-quarter view or all views of the face). They were then asked to construct two composites of that target, one in a full-face view and one in a threequarter view from memory. There were six participants for each of the four targets, ensuring that for every target two participants saw the target in a full-face view, two saw the target in a three-quarter view and two saw all views of the target. As there were six participants each constructing two composites each; one in a three-quarter view and one in a full-face view, this created a total of 48 composites; 12 per target Target order was randomised and construction order was counterbalanced.

\section{Procedure}

The procedure for composite construction was identical to the procedure in stage 1 of Experiment 1. 
Stage 2: Evaluation of Composites

Likeness Ratings

Materials

Each full-face and three-quarter view composite was presented with monochrome photographs depicting the target in both views (one in full-face and one in threequarter view). All images measured $13 \mathrm{~cm}$ in height. The photographs were edited using Microsoft Photo Editor to ensure that brightness and contrast were constant.

\section{Participants}

Twenty-two participants aged between 18 and 45 years were recruited from the University of Stirling. Participants had not taken part in any of the previous experiments and all were unfamiliar with the targets.

\section{Design}

Unfamiliar participants rated the composites for likeness on a scale from one (low) to ten (high). All forty-eight composites were randomly ordered in one presentation book. Each composite was printed on a single sheet of A4 paper and displayed with two monochrome photographs of the target (one full-face and one three-quarter view, printed side by side on a separate sheet of A4 paper). Presentation order was randomised.

\section{Procedure}

This procedure was identical to the likeness rating procedure used in stage 3 of Experiment 1. 


\section{Results and discussion}

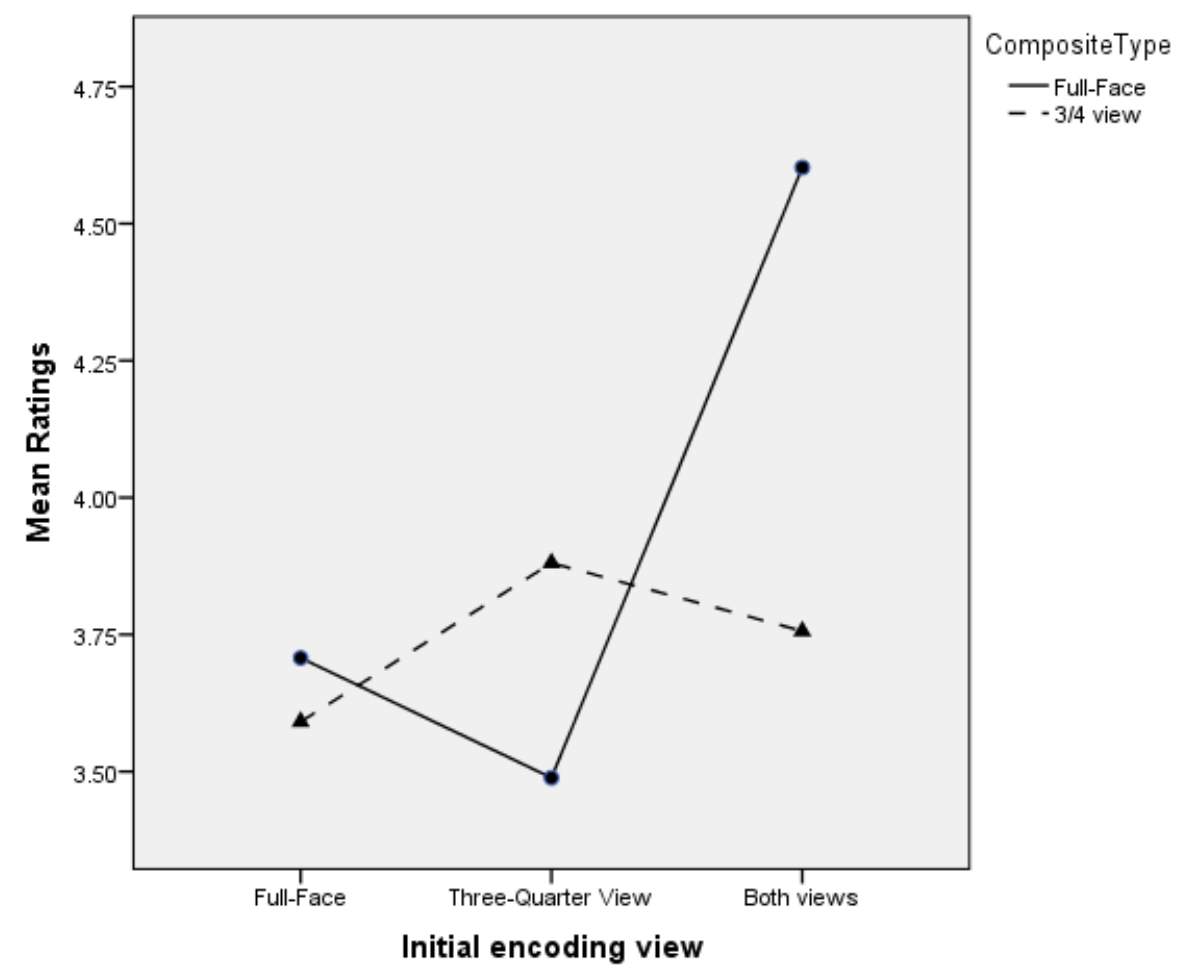

Figure 4: Mean ratings for the full-face and three-quarter view composites by encoding view

A 2 (type of composite; full-face or three-quarter view) by 3 (encoding view; fullface, three-quarter or both views) repeated measures ANOVA was conducted. This revealed a significant main effect of type of composite $[F(1,21)=8.013, p<0.05$, $\left.\bigcap \mathrm{p}^{2} .276\right]$ with higher ratings for the full-face composites $(M=3.9)$ than the threequarter view composites $(M=3.7)$ [ $\mathrm{p}<0.05]$. A significant main effect of encoding view $\left[\mathrm{F}(2,42)=13.676, \mathrm{p}<0.0001,1 \mathrm{p}^{2} .394\right)$ with both views yielding the highest rating scores $(M=4.2)$ compared to full-face $(M=3.6)$ and three-quarter view $(M=3.7)$ [p=0.001 for both]. There was also a significant interaction between composite type and encoding view $\left[\mathrm{F}(2,42)=19.091, \mathrm{p}<0.0001,1 \mathrm{p}^{2} .476\right]$.

Further analysis revealed that the full-face composites were rated as significantly better likenesses when participants had seen all views of the face at encoding $(M=4.6$, $\mathrm{SD}=1.02)$ compared with seeing a full-face view only at encoding $(M=3.7, \mathrm{SD}=1.19)$ or a three-quarter view $(M=3.59, S D=1.22)$ [ $\mathrm{p}<0.0001$ for both respectively]. For the three-quarter view composites a slightly different pattern was observed. The composites were rated as significantly worse likenesses when a full-face view had been seen at encoding $(M=3.4, S D=1.18)$ compared to both the three-quarter view $(M=3.88, S D=1.19)$ and all views $(M=3.75, S D=1.08)$ [ $\mathrm{p}<0.05$ for both respectively]. There was no difference in composite quality when either the three-quarter view or both views had been encoded [p>0.05].

These results do provide some initial support for a moderate encoding specificity effect (e.g. Tulving and Thomson, 1973) . The three-quarter view composites were rated as poorer likenesses when a full-face view had been encoded. Similarly, the 
highest likeness ratings were obtained when the three-quarter view composites had been constructed after encoding the face in a three-quarter view. However, this difference did not quite reach significance. There was no three-quarter view advantage. Instead, when more 3-dimensional information was available at study (either in the form of a three-quarter view or both views) the composites were rated as significantly better likenesses. This also provides support for the symmetry hypothesis (Schyns and Bülthoff, 1994; Troje and Bülthoff, 1996; Hill, Schyns and Akamatsu, 1997; Troje, 1998) and for viewpoint dependency effects in facial composite construction. As it is clear from these results that generalisation to novel views in a composite construction task is dependent on the learning view. Furthermore, the results from the full-face composites suggest that presenting more information at encoding produces better quality composites.

In order to examine this further an additional composite evaluation task was undertaken. A proxy identification task - a 6 alternative forced choice array task was undertaken. This type of task is commonly used in facial composite research (e.g. Bruce, et al.,, 2002).

\section{Stage 2: Array Task \\ Materials}

Target absent and target present arrays were constructed for each of the four targets. The target absent arrays contained monochrome photographs of six similar looking females. The target present arrays contained one monochrome photograph of the target and five distractor photographs. The same distractors were used in both arrays and they were matched visually for hairstyle/colour, face shape and approximate age. All images were standardised for height $(7 \mathrm{~cm})$ and were presented on a single sheet of A4 paper. Microsoft Photo Editor was used to ensure that brightness and contrast were consistent. Four different sets of arrays were constructed (target present full-face view, target present $3 / 4$ view, target absent full-face view and target absent $3 / 4$ view).

\section{Participants}

Two hundred and eighty eight participants aged between 18 and 55 years were recruited from cafeterias and student unions at both the University of Glasgow and Queen Margaret University College, Edinburgh. Participants had not taken part in any of the previous experiments and all were unfamiliar with the targets.

\section{Design}

All 48 composites were presented alone. They were presented with both target present and target absent arrays. View was held constant i.e. three-quarter view composites were presented with three-quarter view arrays and full-face composites were presented with full-face arrays. As composites had been constructed of one target in both views (full-face and three-quarter) these were also presented together. This created a total of 144 presentations (48 single composites and 24 'pairs' of composites presented with both target present and target absent arrays). Careful consideration was given to array view for the pairs. As each pair contained one full-face composite and one three-quarter-view composite, the optimum array would contain both views. However, this was not possible in this experiment and as an advantage for presenting both views had previously been found in experiment one using full-face arrays, these were used. 
To ensure that each participant saw only one composite for each of the four targets, thirty-six separate presentation books were constructed. Each book was balanced for type of composite, initial encoding view and array type and there were 8 participants per book (288 in total).

\section{Procedure}

Participants were told that the composites were constructed from memory and that they represented a likeness of the original target. They were told that when they saw two composites, these represented two views of the same person. Participants were asked to examine all of the images closely and were told that the target may or may not be in the array. They were asked to indicate whether or not they thought the target was in the array. If they thought the target was present, participants were asked to point to the appropriate photograph.

\section{Results and discussion}

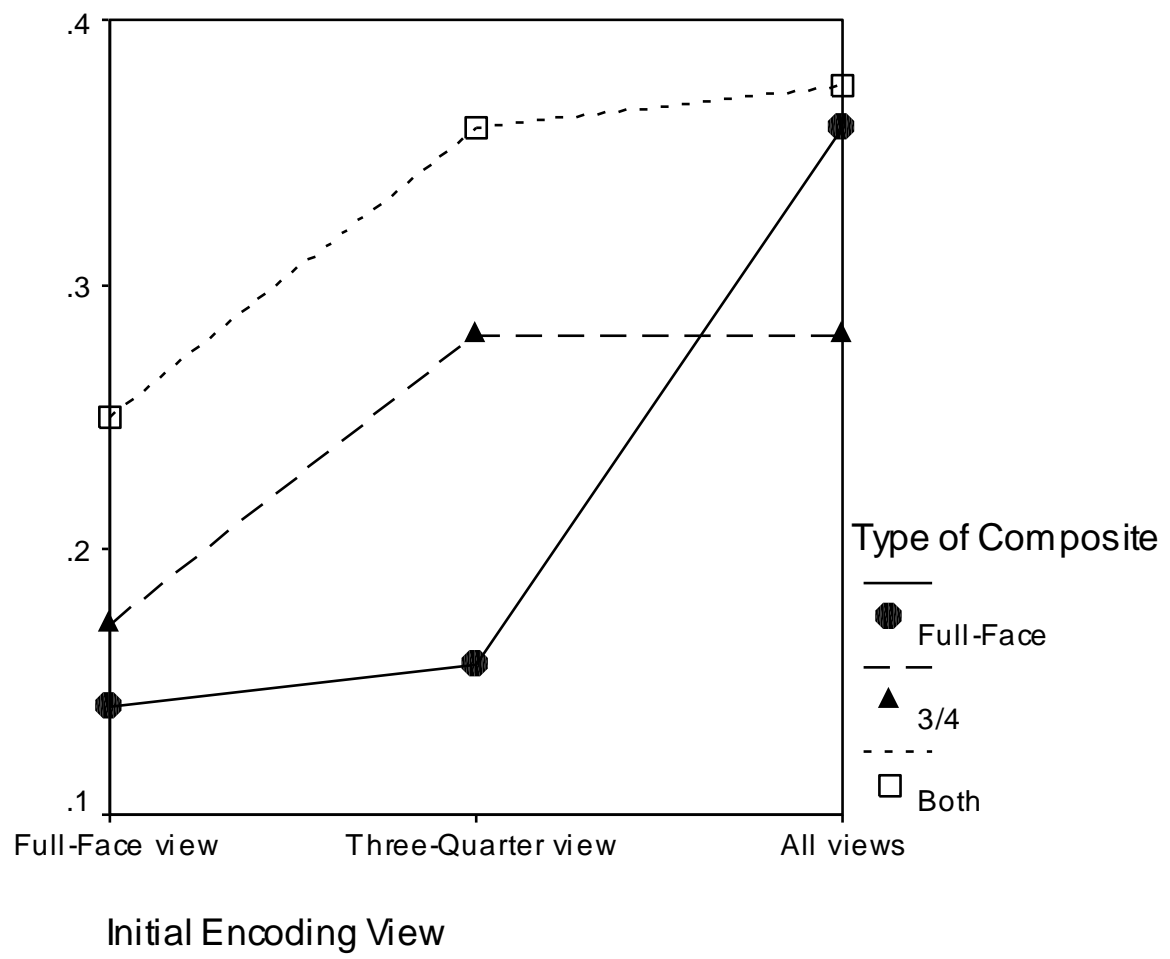

Figure 5: Mean no. of correct matches for full-face composites, three-quarter view composites, both composites presented together, with initial encoding view

The data was initially collapsed across target and encoding view and a Friedman test was conducted on the overall hit rate. This revealed a significant effect $\left[\mathrm{X}^{2}(2)=6.049\right.$, $\mathrm{p}>0.05]$. Further analyses using Wilcoxon Signed Rank tests revealed that when both composites were presented performance was significantly better compared to presenting the single full-face composites [ $p<0.05]$. The presentation of both composites appeared to perform better than the presentation of a single three-quarterview composite, however this difference did not quite reach significance $[\mathrm{p}=0.08]$ ).

A Cochran's Q test on the no of correct matches by type and view revealed significant overall differences $[\mathrm{Q}(8)=22.688, \mathrm{p}<0.05)$. Further analysis on the type of composite 
revealed significant differences for the full-face composites $[\mathrm{Q}(2)=11.806, \mathrm{p}<0.05)$. Pairwise comparisons using Mcnemar tests revealed that there were significantly more correct matches when all views of the face had been encoded compared with both the three-quarter-view encoding $[\mathrm{p}<0.05]$ and the full-face encoding conditions [ $p<0.05]$. No significant differences were observed for the three-quarter-view composites. However the difference between the full-face condition and the threequarter view condition almost reached significance $[p=0.065]$. Similarly, no significant differences were observed when both composites were presented, although the trends are clearly in line with the results from experiments 1 and 2.

Figure 5 displays a similar pattern to the rating data in Figure 4: for the full-face composites, presenting both views of the face at encoding results in better quality composites. For the three-quarter view composites, the quality is poorer when only a full-face has been encoded with no difference between the three-quarter view and both view encoding conditions.

\section{General Discussion}

The results from both the rating and array tasks indicate that a full-face composite will represent a better target likeness when a 'participant witness' has encoded all views of the face. While there is no increase in performance for the three-quarter view composites, performance is still high and there is a marked increase in performance for the full-face composites. The results also suggest that when a 'participant witness' has encoded a side view of a target face, performance will be better when a threequarter-view composite is constructed compared to a full-face composite. Interestingly, the results suggest that when a full-face view has been encoded, performance will be low when a full-face composite is constructed.

The performance of the three-quarter-view composites in the three-quarter encoding condition provides initial support for the encoding specificity principle (e.g. Tulving and Thomson, 1973). However, the performance of the full-face composites does not. Furthermore, the similar performance of the three-quarter-view composites in both the three-quarter and all view encoding conditions indicates that similar information was encoded from both encoding presentations. This appears to provide support for the symmetry argument proposed by Vetter, Poggio and Bülthoff, (1994) who state that learning one view of a bilaterally symmetrical object can be sufficient to generalise to other views. As a face is generally bilaterally symmetrical, then a side view (the symmetrical view), which is non-singular, may contain enough information to generalise to other views (Hill, Schyns and Akamatsu, 1997; Schyns and Bülthoff, 1994). The results from experiment one also support this research by indicating that when all views of a face are presented, no one view is preferred (similar results were obtained by Hill, Schyns and Akamatsu, 1997 \& Schyns and Bülthoff, 1994). However, if the symmetry argument was correct, then performance of the full-face composites should have been higher when a three-quarter-view had been encoded. Performance was slightly higher in the array task, however the results for the ratings task provided initial support for the encoding specificity principle (e.g. Tulving and Thomson, 1973) by indicating that full-face composites were better when a full-face had been encoded. Therefore, the pattern of results obtained for the full-face composites cannot be explained by either the encoding specificity principle or the symmetry argument. 
Previous research has utilised various recognition tasks and the different findings in these experiments may reflect qualitatively different task demands. Composite construction is a reconstruction task where participants need to recall the individual features and then recognise whether the presented feature 'matches' the feature represented in memory. It is unclear at this stage whether this process of recall and recognition is a continuous process, and what effect this has on constructing composites in differing views. Further research needs to be undertaken to examine this.

In addition, Experiments 2 and 3 both suggest that when more information is provided at the identification/evaluation stage, performance increases. This increased performance for presenting two views of a face is only observed when both composites have been constructed, as there is no benefit when one of the composites has been automatically generated. This supports previous research (e.g. Brace et al., 2006; Bruce et al., 2002) by suggesting that the presentation of varied information increases identification. Several experiments (Ness, 2003) have found that simply presenting more information does not serve to increase identification (i.e. the presentation of more than one composite by the same participant in the same view). This may explain the poor performance for presenting a full-face composite with a very similar automatically generated three-quarter-view.

To conclude, the theoretical issues surrounding viewpoint dependency and encoding specificity in a composite construction task need further research. The practical implications of this research however are important. While standard full-face composites using PROfit perform well when all views of the face have been encoded, care should be taken when a person has only seen one view. When a witness has seen a side view of a suspect the results indicate that a three-quarter-view composite should be constructed. In addition, the results also indicate that it would be beneficial for a witness to construct two composites of a suspect, one in full-face view and one in a three-quarter-view, particularly when the witness has only seen one view.

\section{References}

Association of Chief Police Officers and National Police Improvement Agency. (2009), "Facial identification guidance", Available from: http://www.acpo.police.uk/documents/crime/2009/200911CRIFIG01.pdf [Accessed 24th October 2014].

Baddeley, A., and Woodhead, M. (1983), "Improving face recognition ability". In Lloyd-Bostock, S and Clifford, B. (Eds.). Evaluating Witness Evidence. Chichester: Wiley.

Bartlett, F.C. (1932). "Remembering: A Study in Experimental and Social Psychology". Cambridge University Press.

Brace, N.A., Pike, G.E., Kemp, R.I., Turner, J. and Bennett, P. (2006), “Does the presentation of multiple facial-composites improve suspect identification?", Applied Cognitive Psychology. Vol. 20, pp. 213-266.

Bruce, V., Ness, H., Hancock, P.J.B., Newman, C. and Rarity, J. (2002), "Four heads are 
better than one. Combining face composites yields improvements in face likeness", Journal of Applied Psychology, Vol. 87, pp. 894-902.

Bruce, V. \& Valentine, T. (1988). When a nod's as good as a wink. The role of dynamic information in facial recognition. In M. M. Gruneberg \& E. Morris (Eds), Practical Aspects of Memory: Current Research and Issues. Lawrence Erlbaum Associates.

Bruce, V., Valentine, T. and Baddeley, A. D. (1987), "The basis of the $3 / 4$ View advantage in face recognition", Applied Cognitive Psychology, Vol. 1.pp. 109-120.

Cutler, B. L., Stocklein, C. J., \& Penrod, S. D. (1988), “An empirical examination of a computerized facial composite production system", Forensic Reports, Vol. 1. pp. 207218.

Foderella., Kuivaniemi-Smith. and Frowd. (in press), Journal of Forensic Practice

Davies, G., Ellis, H. and Shepherd, J. (1978), "Face identification: The influence of delay upon accuracy of Photofit construction", Journal of Police Science and Administration, Vol. 6, pp. 35-42.

Davies, G., Van Der Willik, P. and Morrison, L. J. (2000), "Facial composite production: A comparison of mechanical and computer-driven systems", Journal of Applied Psychology, Vol. 66, pp. 29-37.

Edelman, S. \& Bülthoff, H. H. (1992), "Orientation dependence in the recognition of familiar and novel views of three-dimensional objects", Vision Research, Vol. 32, pp. 2385-2400.

Fodarella, C., Kuivaniemi-Smith, H., Gawrylowicz, J. and Frowd, C.D. (2015), "Detailed procedures for forensic face construction", Journal of Forensic Practice.

Frowd, C.D., Bruce, V., Ross, D., McIntyre, A. and Hancock, P.J.B. (2007), “An application of caricature: how to improve the recognition of facial composites, Visual Cognition, Vol.15, pp. 1-31.

Frowd, C.D., Carson, D., Ness, H., McQuiston, D., Richardson, J., Baldwin, H. and Hancock, P.J.B. (2005), "Contemporary Composite Techniques: the impact of a forensically-relevant target delay", Legal and Criminological Psychology, Vol. 10, pp. 63-81.

Frowd, C.D., Carson, D., Ness, H., Richardson, J., Morrison, L., McLanaghan, S. and Hancock, P.J.B. (2005), "A forensically valid comparison of facial composite systems", Psychology, Crime and Law, Vol. 11, pp. 33-52.

Frowd, C.D., Pitchford, M., Bruce, V., Jackson, S., Hepton, G., Greenall, M., McIntyre, A. and Hancock, P.J.B. (2010), "The psychology of face construction: giving evolution a helping hand", Applied Cognitive Psychology, Vol. 25, pp. 195-203.

George, B., Gibson, S.J., Maylin, M. I.S. and Solomon, C. J. (2008), "EFIT-V: interactive evolutionary strategy for the construction of photo-realistic facial 
composites", Proceedings of the 10th annual conference on genetic and evolutionary computation, pp. 1485-1490.

Hill, H., Schyns, P. G. and Akamatsu, S. (1997), "Information and viewpoint dependence in face recognition”, Cognition. pp. 201-222.

Koehn, C. E. and Fisher, R. P. (1997), "Constructing facial composites with the Mac A-Mug Pro system", Psychology, Crime and Law, Vol. 3, pp. 209-218.

Kovera, M. B., Penrod, S., Pappas, C. and Thill, D. (1997), "Identification of computer-generated facial composites", Journal of Applied Psychology, Vol. 82, pp, 235-246.

Krouse, F. L. (1981), "Effects of pose, pose change and delay on face recognition performance", Journal of Applied Psychology, Vol. 66, pp. 651-654.

Laughery, K. R., Alexander, J. F. and Lane, A. B. (1971), "Recognition of human faces: Effects of target exposure time, target position, pose position and type of photograph", Journal of Experimental Psychology: Human Perception \& Performance, Vol. 22, pp. 395-416.

Laughery, K. and Fowler, R. (1980), "Sketch artist and identikit procedures for recalling faces”, Journal of Applied Psychology, Vol. 65, pp. 307-316.

Logie, R. H., Baddeley, A. D. and Woodhead, M. M. (1987), "Face Recognition, pose and ecological validity", Applied Cognitive Psychology, Vol. 1, pp. 53-69.

Lui, C. H. and Chaudhuri, A. (2002). Reassessing the $3 / 4$ view effect in face recognition. Cognition. 83.p. 31-48.

McIntyre, A.H., Hancock, P.J.B., Langton, S.H.R. and Frowd, C.D. (unpublished), "The smiling face bias enhances recognition of facial composites".

Memon, A. and Bruce, V. (1983), "The effects of encoding strategy and context change on face recognition", Human Learning: Journal of Practical Research \& Applications, Vol. 2, pp. 313-326.

Memon, A., Meissner, C. A., and Fraser, J. (2010), "The cognitive interview: A metaanalytic review and study space analysis of the past 25 years", Psychology, Public Policy, and Law, Vol 16, pp.340-372.

Ness, H. (2003). (unpublished $\mathrm{PhD}$ thesis), "Improving facial composites produced by eyewitnesses".

Newell, F. N., Chirozo, P. and Valentine, T. (1999), "Recognizing unfamiliar faces: The effects of distinctiveness and view", The Quarterly Journal of Experimental Psychology, Vol. 52A, pp.509-534. 
Palmer, S., Rosch, E. and Chase, P. (1981), "Canonical perspective and the perception of objects", In Long, J. and Baddeley, A. (Eds.). Attention and Performances XI. Lawrence Erlbaum.

Patterson, K. E. and Baddeley, A. D. (1977), "When face recognition fails", Journal of Experimental Psychology: Human Learning and Memory, Vol. 3, pp. 406-417.

Pike, G. E., Kemp, R. I., Towell, N. A. and Phillips, K. C. (1997), "Recognising moving faces: The relative contribution of motion and perspective view information", Visual Cognition, Vol 4, pp. 409-437.

Schiff, W., Banka, L and De Bordes Galdi, G. (1986), "Recognising people seen in events via dynamic "mugshots", American Journal of Psychology, Vol 99, pp.219231.

Schyns, P. G. and Bülthoff, H. H. (1994), "Viewpoint dependence and face recognition", Proceedings of the XVI Meeting of the Cognitive Science Society. pp. 789-793.

Tarr, M. J. and Pinker, S. (1990), "When does human object recognition use a viewercentred reference frame?", Psychological Science, Vol. 1, pp. 253-256.

Thomson, D. M. and Tulving, E. (1970), “Associative encoding and retrieval: weak and strong cues", Journal of Experimental Psychology, Vol. 77, pp.593-601.

Troje, N. (1998), "Generalization to novel views of faces: Psychophysics and models concerning the role of bilateral symmetry", In TaddeiI-Ferretti, C. and Musio, C. (Eds.). Downward Processes in the Perception Representation Mechanisms, World Scientific.

Troje, N. F. and Bülthoff, H. H. (1996), "Face recognition under varying poses: The role of texture and shape", Vision Research, Vol. 36, pp. 1761-1771.

Tulving, E. and Thomson, D. (1973), "Encoding specificity and retrieval processes in episodic memory", Psychological Review, Vol. 80, pp. 352-373.

Wogalter, M. and Marwitz, D. (1991), "Face composite construction: In view and from memory quality improvement with practice", Ergonomics, Vol. 22, pp. 333-343.

Vetter, T., Poggio, T. and Bülthoff, H. H. (1994), "The importance of symmetry and virtual views in three-dimensional object recognition", Current Biology, Vol. 4, pp. $18-23$.

Woodhead, M. M., Baddeley, A. D. and Simmonds, D. C. V. (1979), “On training people to recognise faces", Ergonomics, Vol. 22, pp. 333-343. 